\title{
Behaviour of dogs adopted from an animal shelter
}

\author{
Svatava Vitulová, Eva Voslářová, Vladimír Večerek, Iveta Bedáňová
}

University of Veterinary and Pharmaceutical Sciences Brno, Faculty of Veterinary Hygiene and Ecology, Department of Animal Protection, Welfare and Behaviour, Brno, Czech Republic

Received March 14, 2018

Accepted May 15, 2018

\begin{abstract}
Dog adoption success is influenced by many factors. Current research specifically underlines the importance of good behaviour. In order to collect information on the behaviour of adopted dogs, a questionnaire was handed out to people adopting a dog from one of the Czech shelters. According to the respondents to our survey, $72 \%$ of dogs exhibited behavioural problems in the first week after adoption. The most frequent behavioural problems in adopted dogs were aggression (24\%), fearfulness $(21 \%)$, destructiveness $(17 \%)$, excessive vocalisation $(15 \%)$, and separation anxiety $(13 \%)$. No effect $(P>0.05)$ of sex, age, size or health status was found. However, shelter dogs with a documented history of abuse exhibited problem behaviours after adoption more frequently $(P<0.05)$ than non-abused dogs. The follow-up survey revealed significant $(P<0.001)$ positive changes in the behaviour of dogs six months after leaving the shelter despite a prevailing lack of previous experience among adopters and a lack of professional help. The results suggest that mere patience and time spent in a family instead of the shelter environment can be a solution to at least some of the behavioural problems encountered. We can assume that if professional post-adoption behavioural counselling was provided it could increase the rate of problems solved and decrease the amount of time required for the solution. Professional help may be particularly necessary in the case of aggressive dogs in which no progress has been seen six months after leaving the shelter.
\end{abstract}

Canine, adoption, sociability, aggression, fearfulness

Dog adoption success is influenced by many factors. While the morphology of the dog plays an important role when choosing a shelter dog (e.g. Protopopova et al. 2012; Brown et al. 2013; Voslářová et al. 2015; Žák et al. 2015), the behavioural features become important in the post-adoptive period. The first few weeks after adoption when the dog and the new owner are supposed to be bonding may be critical. According to published data, the proportion of dogs that are returned to the shelter varies from 7\% (Marston et al. 2004) to $17 \%$ (Diesel et al. 2010). Shore (2005) found that more than fifty percent of dogs were returned to the shelter in the first two weeks. Furthermore, half of the returners reported that the problem leading to the return of the animal occurred immediately after the pet was brought home. Several studies have examined the prevalence of behavioural problems during the first months after adoption (New et al. 2000; Wells and Hepper 2000; Marston and Bennett 2003; Shore 2005; Lord et al. 2008). According to Wells and Hepper (2000), the most common problem perceived by adopters of shelter dogs is fearfulness, followed by excessive activity, barking, destructiveness, inappropriate elimination and aggression towards owners, strangers and other animals. Pulling on the leash, separation anxiety, sexual mounting, stealing food, chewing on inappropriate objects, straying, and chasing animals, cyclists or other objects are other exhibited forms of undesirable behaviour (Shore 2005; Bamberger and Houpt 2006; Stephen and Ledger 2007). Duffy et al. (2008) found that problems such as not getting along with 
other pets or children may be difficult to predict. However, behavioural differences can be found among dogs of different sexes and ages. According to Wells and Hepper (2000), male shelter dogs demonstrated more unacceptable behaviours than females; specifically they were more likely than females to exhibit aggression towards other dogs in the first month after adoption. Wells and Hepper (2000) also found adults displaying more inter-dog aggression than juvenile dogs.

A dog's behaviour after adoption may also be affected by the attitude and experience of the new owner. The change of environment may be stressful for the dog especially if the owner lacks knowledge and experience (New et al. 2000). Houpt et al. (1996) pointed out that it is rare to find true cases of problematic behaviours, with these often being the result of a lack of knowledge and understanding on the part of the owner.

The aim of this study was to assess the behaviour of dogs adopted from shelters as perceived by their adopters, to investigate the factors affecting the dog's behavior, and to analyse changes in the dog's behaviour within six months after adoption.

\section{Materials and Methods}

In order to survey adopters we contacted all dog shelters listed on the website of the State Veterinary Administration of the Czech Republic. Of 164 shelters (as of 15 June 2016), 55 shelters responded to our initial enquiry and agreed to participate in the survey, namely, to hand out a questionnaire and an explanatory letter to the persons adopting a dog from their shelter from 1 October 2015 to 31 October 2016. However, questionnaires were provided to all shelters and since we received responses from persons adopting dogs from a total of 84 shelters, it is likely that at least some of those shelters not responding to us made questionnaires available to their adopters.

The questionnaire handed out by the shelters focused on the behaviour of dogs during the first week after adoption. Information regarding whether the respondents' dogs had exhibited any behaviours which they considered unacceptable, and if so what these were, was collected. Furthermore, respondents were required to indicate the prevailing personality traits (aggression, fearfulness, sociability) in dogs in the first week of leaving the shelter. One part of the questionnaire collected information on the demographic characteristics of the adopters (gender, age) and the adopted dogs (sex, age, size, health status), as well as about dog keeping practices (outdoor vs. indoor, presence of children, other dogs or animals in the household) and adopters' level of prior experience with dogs (a high level of experience with dogs including those exhibiting behavioural problems, an ordinary level of experience with dogs, or no previous experience of dog ownership). The questionnaire was anonymous, although respondents were asked if they agreed to be contacted again and were willing to provide their e-mail address. Those who provided an e-mail address were subsequently sent another questionnaire 6 months after adoption. The second questionnaire collected information on the behaviour of dogs 6 months after adoption with the aim of assessing changes in dog behaviour post adoption. Again, respondents were required to indicate the prevailing personality traits (aggression, fearfulness, sociability) in dogs six months after leaving the shelter. Furthermore, respondents were asked if they had sought any help or advice concerning their dog's behaviour and if so where. In the last part of the questionnaire we asked how the adopter's expectations had been met and whether they would adopt a shelter dog again or not.

The results were analysed using the statistical package Unistat 5.6. (Unistat Ltd., London, England). Six independent variables were constructed for the characteristics of the respondents to the survey: gender (2 levels: man, woman); age ( 3 levels: $<25$ years, 25 to 60 years, $>60$ years); the number of persons living in the household (3 levels: one, two, three or more); the presence of children aged up to 10 years in the household ( 2 levels: yes, no); the presence of other animals in the household ( 3 levels: dogs, other animals, no animals); previous experience with dogs (3 levels: none, medium, extensive). Eight independent variables were constructed for the characteristics of the dogs adopted by respondents to the survey: sex ( 2 levels: male, female); age (3 levels: $<2$ years, 2 to 8 years, $>8$ years); size ( 3 levels: small, medium, large); experience of abuse ( 3 levels: yes, no, unknown); dog housing (2 levels: indoor, outdoor); health problems (2 levels: yes, no); behavioural problems (2 levels: yes, no). Firstly, the actual and relative counts (frequencies) of dogs and their adopters in all categories according to the monitored independent variables were calculated and differences between these categories were tested. Frequencies were compared on the basis of a chi-square analysis of $\mathrm{k} \times \mathrm{m}$ and $2 \times 2$ contingency tables. The effect of the dogs' sex, age, size, health status and experience of abuse and the adopters' experience on the prevalence of behavioural problems in dogs was tested and the occurrence of aggression, fearfulness and sociability was compared between the first week and six months after adoption by means of $\mathrm{k} \times \mathrm{m}$ and $2 \times 2$ contingency tables. The age of males and females was compared using Mann-Whitney test. A $P$-value $<0.05$ was considered significant. 


\section{Results}

One hundred and ninety-two persons who had acquired a dog within a 12-month period from 84 shelters in the Czech Republic responded to the survey. Considering the total number of dogs adopted within this period, the response rate varied from 2 to $23 \%$ for individual shelters. All respondents provided an e-mail address and agreed to be contacted with a subsequent survey. However, only 141 of them completed the second questionnaire that was sent to each respondent six months after they adopted a shelter dog. Descriptive characteristics of respondents are given in Table 1 . Significantly $(P<0.001)$ more women responded to the survey. Respondents aged from 25 to 60 years were predominant $(77.6 \%)$. Female respondents were significantly $(P<0.05)$ younger (median age 30 years) than male respondents (median age 42 years). Only $10.4 \%$ of respondents lived in single-person households. Significantly $(P<0.001)$ more respondents shared their household with one or more other persons. Children aged up to 10 years were present in $22.4 \%$ of the respondents' households and animals other than the dog adopted from the shelter were present in $78.1 \%$ of the respondents' households. Most respondents (88.5\%) claimed to have previous experience with dog ownership. However, only $20.3 \%$ of respondents claimed a high level of experience with dogs including those exhibiting behavioural problems.

Table 1. Characteristics of respondents $(\mathrm{n}=192)$.

\begin{tabular}{lrr}
\hline Variable & $\mathrm{n}$ & \multicolumn{1}{c}{$\%$} \\
\hline Owner's gender & & $P<0.001$ \\
$\quad$ Female & 179 & $93.23^{\mathrm{a}}$ \\
Male & 13 & $6.77^{\mathrm{b}}$ \\
Age (years) & 36 & $P<0.001$ \\
$<25$ & 149 & $18.75^{\mathrm{b}}$ \\
$25-60$ & 7 & $77.60^{\mathrm{a}}$ \\
$>60$ & & $3.65^{\mathrm{c}}$ \\
Number of persons in the household & 20 & $P<0.001$ \\
One & 79 & $10.42^{\mathrm{b}}$ \\
Two & 93 & $41.15^{\mathrm{a}}$ \\
Three or more & & $48.44^{\mathrm{a}}$ \\
Presence of children aged up to 10 years in the household & 43 & $P<0.001$ \\
$\quad$ Yes & 149 & $22.40^{\mathrm{b}}$ \\
$\quad$ No & & $77.60^{\mathrm{a}}$ \\
Presence of other animals in the household & 73 & $P<0.001$ \\
Dogs & 77 & $38.02^{\mathrm{a}}$ \\
Other animals & 42 & $40.10^{\mathrm{a}}$ \\
No animals & & $21.88^{\mathrm{b}}$ \\
Previous experience with dogs & 22 & $P<0.05$ \\
None & 131 & $11.46^{\mathrm{c}}$ \\
Medium & 39 & $68.23^{\mathrm{a}}$ \\
Extensive & $20.31^{\mathrm{b}}$ \\
\hline
\end{tabular}

a,b, cPercentages within a column and the same variable with different superscripts differ significantly $(P<0.05)$

Table 2 presents the characteristics of dogs adopted by the respondents to the survey. The ratio of male and female dogs was equal. The median age of both male and female dogs was two years. Dogs of medium size represented almost half of the dogs $(47.9 \%)$ in 
Table 2. Characteristics of dogs $(n=192)$ adopted from shelters by the respondents of the survey.

\begin{tabular}{lcr}
\hline Variable & $\mathrm{n}$ & \multicolumn{1}{c}{$\%$} \\
\hline Sex & & $P>0.05$ \\
Female & 97 & $50.52^{\mathrm{a}}$ \\
Male & 95 & $49.48^{\mathrm{a}}$ \\
Age (years) & & $P<0.001$ \\
$<2$ & 90 & $46.88^{\mathrm{a}}$ \\
$2-8$ & 73 & $38.02^{\mathrm{a}}$ \\
$>8$ & 29 & $15.10^{\mathrm{b}}$ \\
Size & & $P<0.05$ \\
Small & 59 & $30.73^{\mathrm{b}}$ \\
Medium & 92 & $47.92^{\mathrm{a}}$ \\
Large & 41 & $21.35^{\mathrm{c}}$ \\
Experienced abuse & & $P<0.001$ \\
Yes & 79 & $41.15^{\mathrm{a}}$ \\
No & 95 & $49.48^{\mathrm{a}}$ \\
Unknown & 18 & $9.38^{\mathrm{b}}$ \\
Dog housing & & $P<0.001$ \\
Indoor & 177 & $92.19^{\mathrm{a}}$ \\
Outdoor & 15 & $7.81^{\mathrm{b}}$ \\
Health problems & & $P<0.001$ \\
Yes & 27 & $14.06^{\mathrm{b}}$ \\
No & 165 & $85.94^{\mathrm{a}}$ \\
Behavioural problems & & $P<0.001$ \\
Yes & 138 & $71.88^{\mathrm{a}}$ \\
No & 54 & $28.13^{\mathrm{b}}$ \\
\hline
\end{tabular}

a, b,c Percentages within a column and the same variable with different superscripts differ significantly $(P<0.05)$

the study. Small and large dogs were significantly $(P<0.05)$ less numerous. Most dogs $(92.2 \%)$ were housed indoors. Meanwhile health problems were indicated in only $14.1 \%$ of dogs, $71.9 \%$ of dogs exhibited behavioural problems. The most frequent behavioural problems in adopted dogs were aggression $(24.0 \%)$, fearfulness $(21.4 \%)$, destructiveness (16.7\%), excessive vocalisation (15.1\%), and separation anxiety (13.0\%). Fifty-nine dogs exhibited more than one type of problem behaviour. Table 3 summarises specific problem behaviours indicated by respondents in the first week after the adoption of the dog from the shelter. No effect $(P>0.05)$ of sex, age, size or health status was found. However, abuse was found to have a significant $(P<0.05)$ impact on the occurrence of problem behaviours. Shelter dogs with a documented history of abuse exhibited problem behaviours after adoption more frequently than non-abused dogs. Ninety respondents had sought help or advice concerning their dog's behaviour; $39.7 \%$ of them asked friends who were more experienced dog owners, $23.4 \%$ contacted dog trainers, and $17.7 \%$ contacted dog shelter staff.

Figure 1 compares the personality traits in dogs indicated by the respondents in the first week and six months after adoption. No significant $(P>0.05)$ change in the prevalence of aggressive dogs was found after six months. However, the number of fearful dogs significantly $(P<0.001)$ decreased and the number of sociable dogs significantly $(P<0.001)$ increased within the six months after adoption. No effect $(P>0.05)$ of adopters' previous experience with dogs was found on changes to dogs' personality traits. 
Table 3. Specific behavioural problems indicated in dogs exhibiting behavioural problems in the first week after adoption.

\begin{tabular}{lcccc}
\hline \multirow{2}{*}{ Behaviour } & \multicolumn{3}{c}{ Sex } \\
\cline { 2 - 5 } & \multicolumn{2}{c}{ Female $(\mathrm{n}=71)$} & \multicolumn{2}{c}{ Male $(\mathrm{n}=67)$} \\
\hline Aggression & 19 & 26.76 & 27 & $\%$ \\
Fearfulness & 22 & 30.99 & 19 & 40.30 \\
Destructiveness & 20 & 28.17 & 12 & 28.36 \\
Excessive vocalisation & 16 & 22.54 & 13 & 17.91 \\
Separation anxiety & 15 & 21.13 & 10 & 19.40 \\
Inappropriate elimination & 13 & 18.31 & 6 & 14.93 \\
Escapes & 6 & 8.45 & 4 & 8.96 \\
Jumping on people & 1 & 1.41 & 5 & 5.97 \\
Self-harming & 0 & 0.00 & 2 & 2.46 \\
Pulling on the leash & 1 & 1.41 & 1 & 1.49 \\
\hline
\end{tabular}

Overall, $96.5 \%$ of respondents declared that they would adopt an animal from a shelter again and $81.6 \%$ of respondents claimed that they did not face any unexpected difficulties related to the adoption of a shelter dog.

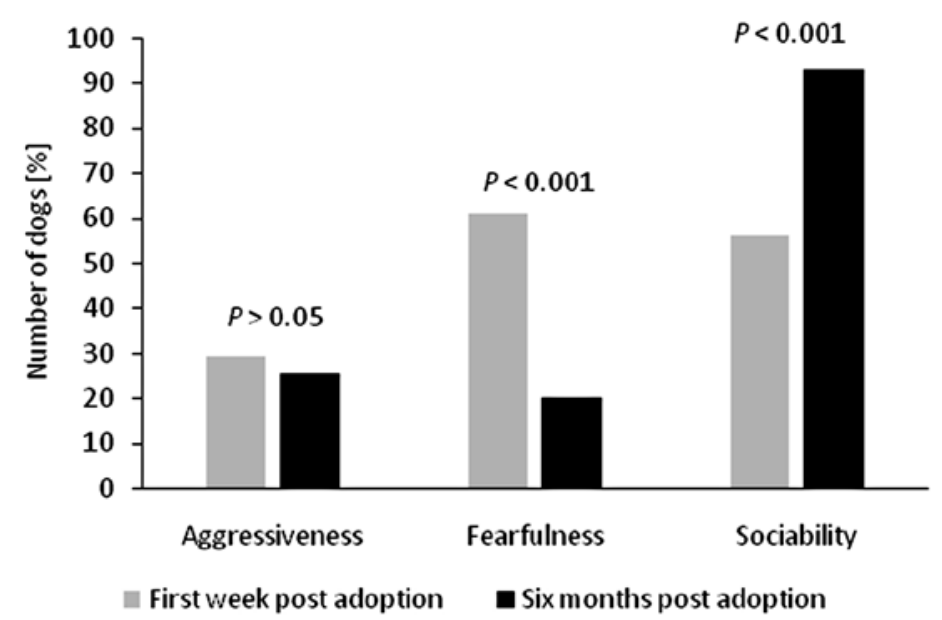

Fig. 1. Comparisons of prevailing personality traits in dogs $(n=141)$ as indicated by their adopters in the first week and six months after adoption.

\section{Discussion}

Once a dog has been adopted by a new owner, it needs to be integrated into its new home. Ideally, a strong relationship develops between dogs and their owners, but the maintenance of this bond is not an obvious consequence; many events can jeopardise its success and length (Mondelli et al. 2004). Current research underlines the importance of good behaviour to dog adopters specifically (O'Connor et al. 2016). However, the majority $(68 \%)$ of respondents who had purchased a dog from a rescue shelter in Northern Ireland (Wells and Hepper 2000), as well as those who adopted a dog from the Michigan Humane Society in the USA (Lord et al. 2008), reported that their dog exhibited 
a behavioural problem. Similarly, $72 \%$ of newly adopted dogs exhibited behavioural problems according to the respondents to our survey. Given how common the presence of problem behaviour in shelter dogs is and how often it was cited as the primary reason for relinquishment or returning dogs to an animal shelter (e.g. Miller et al. 1996; DiGiacomo et al. 1998; Salman et al. 1998; Wells and Hepper 2000; Mondelli et al. 2004), providing help to adopters to remedy common behavioural problems in shelter dogs could significantly increase the rate of successful dog adoptions. Access to appropriate behavioural advice and post-adoptive obedience training may not merely improve the dogs' behaviour but also re-align the owner's expectations of normal canine behaviour (Patronek et al. 1996). Marston and Bennett (2003) point out that it is unclear whether behavioural problems reported by new adopters reflect 'real' behavioural problems or a mismatch between 'normal' dog behaviour and unrealistic expectations on the part of the new owner. However, adoption success depends on how the dog is perceived by its new owner and therefore, all issues compromising the dog-owner relationship need to be addressed. The respondents to our study considered aggression, fearfulness, destructiveness, excessive vocalisation, separation anxiety, escapes, jumping on people, self-harming and pulling on the leash as problem behaviours. This list corresponds to the findings of studies that examined the prevalence of behavioural problems in dogs in the UK (Wells and Hepper 2000; Stephen and Ledger 2007) and the USA (Shore 2005; Bamberger and Houpt 2006). In contrast to Wells and Hepper (2000), who reported the effect of sex and age on the prevalence of behavioural problems, no such differences were found in our study. Behavioural problems were equally reported in adopted dogs of both sexes, different ages and size categories. Nor did any other variable analysed in our study affect the prevalence of behavioural problems, with the exception of experience of animal abuse. Shelter dogs with a documented history of abuse exhibited problem behaviours after adoption more frequently than non-abused dogs. Accordingly, McMillan et al. (2015) reported that canine victims of animal abuse displayed significantly higher rates of aggression and fear directed toward unfamiliar humans and dogs, excitability, hyperactivity, attachment and attention-seeking behaviours, persistent barking, and miscellaneous strange or repetitive behaviours when compared with non-abused dogs. For the purposes of their study, dogs with a known or suspected history of abuse were solicited. Interestingly, $75.4 \%$ of them had been acquired from a shelter or rescue group. In our study, $41.2 \%$ of dogs adopted from shelters by the respondents to our survey had a history of abuse. The real ratio may be even higher as the history of some dogs was unknown. The results indicate that when adopting a shelter dog, there is a high probability that it has been abused in the past and will manifest some kind of problem behaviour which is all the more reason to propose post-adoption behavioural counselling provided by professionals as routine practice. Despite the high occurrence of behavioural problems and limited previous experience with dogs, only a small number of the adopters in our study sought professional advice.

A trait-based approach was taken in order to measure general personality traits and their changes post-adoption. For dogs, this takes the form of informant-based techniques that rely on an informed observer (the dog's owner) to recall and report the dog's behaviour, synthesising their experience with the dog. Informant-based techniques have demonstrated a good degree of inter-observer reliability and may actually be less subjective than behaviourally-based techniques (Dowling-Guyer et al. 2011; Patronek and Bradley 2016). Despite low associations between results in behaviour tests and the owners' perception of the dogs' everyday behaviour (Svartberg 2005), dog behaviour has previously been studied mainly by analysing results from behaviour tests, working trials, etc. and not everyday behavioural data (Eken Asp et al. 2014). Dowling-Guyer et al. (2011) investigated behavioural traits in shelter dogs and specifically identified aggression, fearfulness and sociability (often called friendliness). These traits are broadly defined, 
readily interpretable and consistent with other research (Jones and Gosling 2005; Svartberg et al. 2005; Eken Asp et al. 2014). Most potential dog owners are looking for a non-aggressive, non-fearful, social and easily trained dog that functions well in everyday life (Eken Asp et al. 2014). The behaviour of shelter dogs as reported by their new owners in the first week after adoption in our survey may be discouraging for potential adopters. One third of dogs were reported to be aggressive, almost two-thirds were fearful, and only half were reported to be social. However, despite the above-mentioned general lack of previous experience and professional help, major positive changes in the dogs' behaviour were seen six months after leaving the shelter. The number of fearful dogs decreased ( $61 \%$ vs $20 \%$ ), whereas the number of sociable dogs increased (56\% vs $93 \%$ ) within the six months of adoption. Fearfulness is extremely common amongst adopted dogs in the first weeks of acquisition (Wells and Hepper 2000). Moreover, fearful dogs were reported to be returned to the shelter more often than normal dogs (Normando et al. 2006). However, our results suggest that the intensity of fearful and social behaviours manifested shortly after adoption does not necessarily reflect the normal disposition of the dog, and may change over time. Similarly, Wells and Hepper (2000) concluded that the period of time spent in the shelter environment, albeit relatively short, induced fears in some of the dogs in their study. Furthermore, Shin and Shin (2017) found a relationship between sociability toward humans and physiological stress in dogs. The transition from a shelter to a new home is always a considerable change and dogs need time to adjust. Unfortunately, many dogs are returned within the first two weeks of adoption (Mondelli et al. 2004; Shore 2005; Diesel et al. 2008), i.e. before they can settle into the new environment. One issue that should be addressed by the adopters is aggression. No important change was found in the prevalence of aggression over the course of six months in our study. Dogs exhibiting aggression would therefore seem to be less likely to change their behaviour without appropriate intervention.

Overall, the majority of respondents declared that they would adopt a dog from a shelter again and claimed that they did not face any unexpected difficulties related to the adoption of a shelter dog. The respondents to a survey carried out in the USA by McMillan et al. (2015) rated their adoption experience equally high, $96.2 \%$ of them declaring to be extremely satisfied and $100 \%$ of them stating that they would adopt the same dog again despite knowing what they know now, i.e. the necessity of dealing with problem behaviours. Similarly, Neidhart and Boyd (2002) reported that most adopters were satisfied with their adoption experiences and their adopted animals. Altogether, the results are encouraging. However, these numbers may be biased if the satisfaction level was low in the adopters who did not elect to participate in the surveys. Due to the absence of personal data of all adopters we can only assume that the survey respondents provide a representative sample. This limitation applies to the results of all published adoption surveys. It is, however, noteworthy that the characteristics of respondents to our survey correspond with the characteristics of adopters responding to adoption surveys carried out in different countries (e.g. Neidhart and Boyd 2002; Mondelli et al. 2004; McMillan et al. 2015; O'Connor et al. 2016).

In conclusion, the majority of newly adopted dogs exhibited behavioural problems and personality traits usually perceived as undesirable. However, despite the prevailing lack of previous experience and, in most cases, no professional help provided, major positive changes in the dogs' behaviour were reported six months later. The results suggest that mere patience and time spent in the family instead of the shelter environment may be a solution to at least some behavioural problems. We can assume that if professional post-adoption behavioural counselling was provided it could increase the rate of problems solved and decrease the length of time required for their solution. Professional help may be particularly necessary in the case of aggressive dogs in which no progress has been seen six months after leaving the shelter. 


\section{References}

Bamberger M, Houpt KA 2006: Signalment factors, comorbidity, and trends in behavior diagnoses in dogs: 1,644 cases (1991-2001). J Am Vet Med Assoc 229: 1591-1601

Brown WP, Davidson JP, Zuefle ME 2013: Effects of phenotypic characteristics on the length of stay of dogs at two no kill animal shelters. J Appl Anim Welf Sci 16: 2-18

Diesel G, Brodbelt D, Pfeiffer DU 2010: Characteristics of relinquished dogs and their owners at 14 rehoming centers in the United Kingdom. J Appl Anim Welf Sci 13: 15-30

Diesel G, Pfeiffer DU, Brodbelt D 2008: Factors affecting the success of rehoming dogs in the UK during 2005. Prev Vet Med 84: 228-241

DiGiacomo N, Arluke A, Patronek GJ 1998: Surrendering pets to shelters: the relinquishers perspective. Anthrozoos 11: 41-51

Dowling-Guyer S, Marder A, D'Arpino S 2011: Behavioral traits detected in shelter dogs by a behavior evaluation. Appl Anim Behav Sci 130: 107-114

Duffy DL, Hsu Y, Serpell JA 2008: Breed differences in canine aggression. Appl Anim Behav Sci 114: 441-460

Eken Asp H, Arvelius P, Fikse WF, Nilsson K, Strandberg E 2014: Genetics of aggression, fear and sociability in everyday life of Swedish dogs. In: Proceedings of the 10th World Congress of Genetics Applied to Livestock Production. Vancouver, Canada, no. 795

Houpt KA, Honig SU, Reisner IR 1996: Breaking the human-companion animal bond. J Am Vet Med Assoc 208: 1653-1659

Jones AC, Gosling S 2005: Temperament and personality in dogs (Canis familiaris): A review and evaluation of past research. Appl Anim Behav Sci 95: 1-53

Lord LK, Reider L, Herron ME, Graszak K 2008: Health and behavior problems in dogs and cats one week and one month after adoption from animal shelters. J Am Vet Med Assoc 233: 1715-1722

Marston LC, Bennett PC 2003: Reforging the bond-towards successful canine adoption. Appl Anim Behav Sci 83: $227-245$

Marston LC, Bennett PC, Coleman GJ 2004: What happens to shelter dogs? An analysis of data for 1 year from three Australian shelters. J Appl Anim Welf Sci 7: 27-47

McMillan FD, Duffy DL, Zawistowski SL, Serpell JA 2015: Behavioral and psychological characteristics of canine victims of abuse. J Appl Anim Welf Sci 18: 92-111

Miller DD, Staats SR, Partlo C, Rada K 1996: Factors associated with the decision to surrender a pet to an animal shelter. J Am Vet Med Assoc 209: 738-742

Mondelli F, Previde EP, Verga M, Levi D, Magistrelli S, Valsecchi P 2004: The bond that never developed: Adoption and relinquishment of dogs in a rescue shelter. J Appl Anim Welf Sci 7: 253-266

Neidhart L, Boyd R 2002: Companion animal adoption study. J Appl Anim Welf Sci 5, 175-192

New JC, Salman MD, King M, Scarlett JM, Kass PH, Hutchison JM 2000: Characteristics of shelter-relinquished animals and their owners compared with animals and their owners in U. S. pet-owning households. J Appl Anim Welf Sci 3: 179-201

Normando S, Stefanini C, Meers L, Adamelli S, Coultis D, Bono G 2006: Some factors influencing adoption of sheltered dogs. Anthrozoos 19: 211-224

O'Connor R, Coe JB, Niel L, Jones-Bitton A 2016: Effect of adopters' lifestyles and animal-care knowledge on their expectations prior to companion-animal guardianship. J Appl Anim Welf Sci 19: 157-170

Patronek GJ, Bradley J 2016: No better than flipping a coin: Reconsidering canine behavior evaluations in animal shelters. J Vet Behav 15: 66-77

Patronek GJ, Glickman LT, Beck AM, McCabe GP, Ecker C 1996: Risk factors for relinquishment of dogs to an animal shelter. J Am Vet Med Assoc 209: 572-581

Protopopova A, Gilmour AJ, Weiss RH, Shen JY, Wynne CDL 2012: The effects of social training and other factors on adoption success of shelter dogs. Appl Anim Behav Sci 142: 61-68

Salman MD, New, JG, Scarlett JM, Kass PH, Ruch-Gallie R, Hetts S 1998: Human and animal factors related to the relinquishment of dogs and cats in 12 selected animal shelters in the United States. J Appl Anim Welf Sci 1: $207-226$

Shin YJ, Shin NS 2017: Relationship between sociability toward humans and physiological stress in dogs. J Vet Med Sci 79: 1278-1283

Shore ER 2005: Returning a recently adopted companion animal: adopters' reasons for and reactions to the failed adoption experience. J Appl Anim Welf Sci 8: 187-198

Stephen J, Ledger R 2007: Relinquishing dog owners' ability to predict behavioural problems in shelter dogs post adoption. Appl Anim Behav Sci 107: 88-99

Svartberg K 2005: A comparison of behaviour in test and in everyday life: evidence of three consistent boldnessrelated personality traits in dogs. Appl Anim Behav Sci 91: 103-128

Svartberg K, Tapper I, Temrin H, Radesäter T, Thorman S 2005: Consistency of personality traits in dogs. Anim Behav 69: 283-291 
Voslářová E, Žák J, Večerek V, Bedáňová I 2015: Breed characteristics of abandoned and lost dogs in the Czech Republic. J Appl Anim Welf Sci 18: 332-342

Wells DL, Hepper PG 2000: Prevalence of behaviour problems reported by owners of dogs purchased from an animal rescue shelter. Appl Anim Behav Sci 69: 55-65

Žák J, Voslářová E, Večerek V, Bedáňová I 2015: Sex, age and size as factors affecting the length of stay of dogs in Czech shelters. Acta Vet Brno 84: 407-413 